\title{
The Association between Serum Lipid Profile and Retinopathy in Type 2 Diabetes Mellitus Patients
}

\author{
Karan VN ${ }^{1}$, Vijaya Mohan ${ }^{2}$ \\ ${ }^{1}$ Senior Resident, Department of General Medicine, Mahaveer Institute of Medical Sciences, Vikarabad, Telangana, ${ }^{2}$ Associate Professor, Department of \\ General Medicine, KBNIMS, Gulbarga, Karnataka.
}

\section{Abstract}

Background: Blood such as VLDL, intermediate density lipoprotein (IDL), chylomicrons, chylomicron remnants and lipoprotein and therefore provides a more accurate estimate of CV risk than LDL-C alone, as shown in numerous epidemiological and clinical studies. This is particularly true for patients who are already on statin therapy or those with elevated levels of VLDL-C such a patients with obesity, metabolic syndrome and diabetes. Subjects and Methods: A pre-structural proforma was used to collect baseline date detailed clinical history with clinical examination and relevant investigation was done on participating individuals. Results: Significant correlation between HbA1c and various circulating lipid parameters and significant difference of lipid parameters in two groups $(</ .0 \%$ and $>7.0 \%)$ of glycated haemoglobin indicates that $\mathrm{HbA} 1 \mathrm{c}$ can be used as a potential biomarker for predicting dyslipidemia in type 2 diabetic patients. Conclusion: As good control of diabetes is shown to keep the lipid levels in near normal range, it appears important to aim at critical control of DM to prevent or at least postpone the onset of various complications.

Keywords: Dyslipidemia, Hba1c, Lipid Parameters.

Corresponding Author: Dr. Vijaya Mohan, Associate Professor, Department of General Medicine, KBNIMS, Gulbarga, Karnataka.

Received: June 2019

Accepted: June 2019

\section{Introduction}

A typical lipid profile consists of TG. TC, HDL-C and LDLC. Most of the laboratories measure TC, TG and HDL-C directly using enzymatic assays but LDL-C is often derived indirectly form the Friedewald equation (LDL-C $=$ TC-HDLC-TTG/5). As feeling acutely affects serum TG levels, a fasting blood sample (after 9-12h fasting) is required to estimate LDL-C accurately. This presents a practical challenge as many-a-times circumstances do notallow fasting sampling. In such settings, using non-HDL-C instead of LDL-C is a good alternative. Non-HDL-C is calculated simply by subtracting HDL-C remains accurate irrespective of fasting status. As discussed subsequently, non-HDL-C has several additional advantages over LDL-C as a CV risk marker. ${ }^{[1]}$

Blood such as VLDL, intermediate density lipoprotein (IDL), chylomicrons, chylomicron remnants and lipoprotein (a) $\{L p(a)\}$ and therefore provides a more accurate estimate of $\mathrm{CV}$ risk than LDL-C alone, as shown in numerous epidemiological and clinical studies. This is particularly true for patients who are already on statin therapy or those with elevated levels of VLDL-C such a patients with obesity, metabolic syndrome and diabetes.

Some prospective studies have reported that non-fasting serum triglyceride levels may be a useful predictor of CV events. However, issues such as standardizing sampling conditions and reference values are yet to be clarified.
It is a major risk factor for CVD and increases the risk of CVD by five times in women's, and three fold in men. It has been demonstrated that people with diabetes and no history of MI have a CV risk nearly equivalent to those without diabetes and a history of MI. The mechanisms postulated to cause rapid development of vascular disease in diabetes include hyperglycaemia. Hypertension, low HDL-C, high triglyceride levels, elevated small-dense LDL, increased procoagulant activity and a pro-inflammatory milieu. It stands to reason, therefore, that lipid management is an essential part of diabetes care. ${ }^{[2]}$

As mentioned above, people with diabetes are classified as high risk patients for vascular events. Hence, irrespective of the presence of absence of to her risk factors on history (age, gender, smoking, hypertension, family history) or physical examination (obesity, hypertension, polycystic ovary syndrome in woman), they should be screened for dyslipidemia.

A fasting lipid profile should be performed annually. The minimum investigations should include TC, LDL-C, TG, and HDL-C. Non-HDL-C should be routinely calculated in these patients given the higher prevalence of elevated triglycerides and small-dense DL among diabetics. In addition, estimation of Appo-B levels is also desirable in these patients. However, routine assessment of these markers is not mandatory.

As all patients with type 2 diabetes with evidence of target organ damage or other $\mathrm{CV}$ risk factors and those with type 1 
diabetes with micro albuminuria are automatically designated to high risk category, they require management which is similar to that for secondary preventing of CVD. In other patients, formal risk assessment may be needed. The treatment approach is broadly same as that for any high risk individual without diabetes, as described in the previous sections. The primary target is LDL-C, while non HDL-C, HDl-C and apo B are secondary targets. The present document reiterates the fact that these goals are global, i.e. for both genders and for all adults. However, in children and adolescents, the acceptable LDL-Clel is relaxed to 110 $\mathrm{mg} \%$. ${ }^{[3]}$

Management of dyslipidemia in diabetes is similar to that in people without diabetes. Non-pharmacological therapy viz physical activity, cessation of smoking, and healthy nutrition therapy are important aspects of therapy and should follow the same principles as outlined in the previous sections. However, it should be noted that dietary fructose leads to hypertriglyceridemia if consumed in excess of $10 \%$ of total. ${ }^{4}$ The choice of drug therapy is similar in dyslipidemic persons with and without diabetes. The present guidelines strongly recommend stain therapy despite the fact that certain studies document a rise in incidence of diabetes with these drugs. Meta-analysis has shown that stain use in linked to higher( $9 \%$ ) risk of development of new-onset diabetes especially in older persons. However, the risk: benefit ratio of statins is strongly tilted in favour of drug use.

Although the choice of stain in diabetes is broadly similar to that in people without diabetes, there are some important differences in the impact of different statins on the glycemic control. Glucose neutral effects have been reported for pravastation. Simvastation has been show to inhibit glucoseinduced insulin secretion through blockade of L-type $\mathrm{Ca}^{2+}$ channels in B cells. Atorvastation is thought to suppress glucose transporter GLUT4 expression in 3T3-L1 adipose cells by blocking isoprenoid synthesis. Cytotoxic effects on the B cell have also .been reported for atorvastation. Another postulated mechanism is through activation of SREBPs (Sterol Regulatory Element-Binding Proteins). Pitavastationuse'has been reported to be devoid of the adverse effects on glycaemia that are reported with atorvastatin. Pitavastatin, in fact demonstrated $\mathrm{t}$ beneficial effect on HbA1c in subjects with diabetes who were enrolled in the LIVES study. Differences in the metabolic pathways for various statins may explain these differential effects on glycemicControlpitavastatin is minimally metabolized by the CYP3A4 isoenzyme, unlike other statins, and this may be responsible for its glucose-neutral character. ${ }^{[5]}$

The patients with diabetes often have multiple co-morbidities and are recognized to be in a 'poly-medicated' state. Therefore, lipid therapy in diabetics should have low risk of drug-drug interactions. While most statins are safe, one should be aware of potential drug-drug interactions. Afntifungal agents such as itraconazole, commonly prescribed in diabetes, may increase atorvastatin and simvastatin concentrations' by inhibiting CYP3A4, which metabilizes these statins.

Fenofibrae is the most widely used fibrae compound, and is recommended for use as add-on to statins. Addition of fenofibrate to statin therapy may benefit patients with diabetes, hyperglyceridemia and low HDL-C.Gemfibrozil can also be used in patients with TG>200mg\% and HDL-
$\mathrm{C}<40 \mathrm{mg} \%$, who do not respond to statin monotherapy. However, it does not offer any advantages as compared to fenofibrate. Monotherapy with fibrates is suggested only in patients with isolated hypertriglyceridemia who do not tolerate statin therapy, even at low doses. ${ }^{[6]}$

\section{Subjects and Methods}

A pre-structural proformawas used to collect baseline date detailed clinical history with clinical examination and relevant investigation was done on participating individuals.

\section{Exclusion Criteria}

- $\quad$ Patient with liver disorders

- Patients with $\mathrm{BMI}>30 \mathrm{Kg} / \mathrm{m} 2$

- Patients on lipid lowering agents (statins, fibrates, etc.)

- Patients on treatment with medications which alter lipid profile

- Patients with age $<20$ years and $>80$ years.

- Any situation that shortens erythrocytes survival or decreases mean erythrocyte age falsely lowers $\mathrm{GHb}$ (HbA1c) test results regardless of the test method.

- Iron deficiency anaemia is reported to increase test results that can subsequently be reversed by iron treatment. Hemolyticanemia has opposite effect to iron deficiency by reducing Hbalc in affected individuals.

- It has been reported that results may be inconsistent in patients who have conditions such as opiate addiction, lead poisoning, and alcoholism, ingest large doses of Aspirin.

- HbA1c assays are not recommended for use in the diagnosis of diabetes mellitus.

- It is reported that antibodies used in immuoturbidimetric assays recognize the $\mathrm{N}$-terminal glycated amino acid in the context of 4-10 amino acids of the $\mathrm{Hb}$ b-chain. These antibodies do not recognize the reversible Schiff base (aldimine) or 6 other gHb species, including chemically modified derivatives.

- $\mathrm{HbE}$ has little effect on the determination of glycosylated haemoglobin with immunoassay methods.

- It has been documented that HbF has little or no immunoreactivity with most antibodies used in $\mathrm{HbA1c}$ assays.

- Any patient with a significant change in Hba1c coinciding with a change in laboratory HbA1c methods should be evaluated for the presence of variant or derivative $\mathrm{Hb}$.

- All Hba1c methods are inadequate for the assessment of long-term glycemic control in patients homozygous for $\mathrm{HbS}, \mathrm{HbC}$ or HbSC disease

\section{Results}

Table 1: Sex with Retinopathy
\begin{tabular}{|l|l|l|l|}
\hline Gender & Retinopathy & Total \\
\hline & Absent & Present & \\
\hline Female & $11(28.20 \%)$ & $28(71.79 \%)$ & $39(100 \%)$ \\
\hline Male & $14(22.95 \%)$ & $47(77.04 \%)$ & $619100 \%)$ \\
\hline Total & $25(25 \%)$ & $75(75 \%)$ & $100(100 \%)$ \\
\hline
\end{tabular}




\begin{tabular}{|l|l|l|l|}
\hline Table 2: Age with Retinopathy \\
\hline Gender & Retinopathy & Total \\
\hline & Absent & Present & \\
\hline$<+35$ & 2 & 1 & 3 \\
\hline $36-45$ & 5 & 5 & 10 \\
\hline $46-55$ & 11 & 24 & 35 \\
\hline $56-65$ & 5 & 31 & 36 \\
\hline $66+$ & 2 & 14 & 16 \\
\hline Total & 25 & 75 & 100 \\
\hline
\end{tabular}

Mean + SD: $57.29+11.144$

features of diabetic dyslipidemia are a high plasma triglyceride concentration, low HDL cholesterol concentration and increased concentration of small dense LDL-cholesterol particles. The lipid changes associated with diabetes mellitus are attributing to increased free fatty acid flux secondary to insulin resistance. The availability of multiple lipid-lowering drugs and supplements provides new opportunities for patients to achieve target lipidlevela.

A study in Allahabad concluded that Hypercholesterolemia, hypertri-glyceridaemia and lipoprotein are the main lipid abnormalities found in diabetes which is risk forcoronary artery disease. In diabetes sex plays a signification effect on risk of coronary artery disease.

Present study showed common lipid abnormalities during diabetes induced dyslipidemia are hypercholesterolemia hypertriglycerdemia and elevated LDL-C results suggest a high prevalence of dyslipidemia, which might be playing a major role in the development of cardiovascular disease among diabetic patients. The optimal care of diabetic patients should include routine monitoring of blood sugar and serum lipid profile. Aggressive lifestyle changes, such as weight reduction and physical exercise should be initiated first followed by medication with lipid loweringdrugs. The optimum treatment with antidiabetic drugs to obtain fair glycaemic control should go hand -=in-hand with lipidlowering drugs. ${ }^{[10]}$

It was found that TG and TG/HDL-C were independent DM risk factors, with the odds ratios being $1.292(\mathrm{P}=0.47)$ and 1.341 (p-.010), respectively although they were poor in their DM discriminatory power (area under the receiver operating characteristic curve, 0.662 and 0.672 , respectively). Combined with other risk factors (fasting plasma glucose, waist circumference, and family history of DM), the DM discriminatory power of TG and TG/HDL-C was improved (area under the receiver operating characteristic curve, 0.764 and 0.767 , respectively). The DM incidence increased with ascending risk score. Single HDL-C seems unable to predict future DM. Triglycerides and TG and TG/HDL-C were independent DM risk factors; and of the two TG and TG/HDL-C was a stronger risk factor. The DM discriminatory power of TG and TG/HDL-C was poor; therefore, it is recommended that they he used in combination with oter risk factors. Diabetes mellitus incidence increased with ascending risk score.

Majority of the type 2 diabetes mellitus patients in Aftrica do not maintain a good glycemic control. Diabetic dyslipidemia characterized by high plasma triglycerides, high LDLcholesterol and low HDL-cholesterol is tightly associated with glycemic control. Good glycemic control could result in improvement in the lipid profile and the patients could be spared from the high cardiovascular risk. Combination therapy is better than mono-therapy in controlling the glycemic load in type 2 diabetes mellitus. Metformin added to the other hypoglycaemic drugs gives added benefit in the form of reduced glycemic load and improvement in the lipid profile.

A study in Yemen concluded The overall prevalence of type II diabetes Mellitus was $4.6 \%$ (7.4\% in males and $2 \%$ in females). 1mpaired glucose tolerance (LGT) and impaired fasting glucose (IFG) were found in $2 \%$ and $2.2 \%$ of the study population. Factors independently related to any abnormality in glucose tolerance, using logistic regression 
analysis, were sex, hyperlipidaemia, hypertriglyceridaemia, and hypertension; whereas sex and age related to DM. More than $80 \%$ of the type II diabetics were over the age of 40 , $35 \%$ being hyperlipidaemic, $22 \%$ being hypertensive and $18 \%$ obese. Sixty percent of IGT subjects were hyperlipidaemic and $20 \%$ were obese. Approximately $78 \%$ of obese individuals $(>/=30 \mathrm{~kg} / \mathrm{m}(2))$ had normal glucose tolerance. $^{[11]}$

With comparable glycemic control, the fixed PM (Pioglitazone +metformin) combination was more efficacious on HDL cholesterol improvement than the GM (glmipride+metformin) combination. Additional positive effects were observed forbiomarkers of lipid metabolism, Bcell function, activity of the visceral adipose tissue, and chronic systemic inflammation.

It is concluded from the results of the present study that type 2 diabetics were either overweight or type I obese and dyslipidaemia was very common. Results strongly suggest that further investigations should related the effects of dyslipidaemia and abnormalities of insulin resistance in type 2 diabetics. And ethnic specific patterns of lipid profile in type 2 diabetics regardless of their glucose levels, suggesting that ethnic specific strategies and guidelines on risk assessment and prevention of CVD due to dyslipidemia are required. ${ }^{[12]}$

\section{Conclusion}

Diabetic patients with complications tend to have higher levels of lipid fractions (TGs, cholesterol, and LDL-C) and lower levels of HDL-c. This suggests that there appears to be some relation between the genesis of various vascular complications (micro vascular and macro vascular), and the presence of lipid abnormality. It is difficult to point out a particular factor as the cause as multiple mutually interacting factors determine the presence or development of these complications.

\section{References}

1. Bhardwaj S., Mistra A., Misra R. High prevalence of abdominal, intraabdominal and subcutaneous adiposity and clustering of risk factors among urban Asian Indians in North India. PLos One. 2011;6:e24362.

2. Bansal M., Shrivastava S., Mehrotra R., Agrawal V., Kasliwal R.R. Time trends in prevalence and awareness of cardiovascular risk factors in an asymptomatic North Indian urban population. J Assoc Physicians India. 2009;57:568-573.

3. KasliwalR.r., Kulshreshtha A., Agrawal S., Bansal M., Trehan N. Prevalence of cardiovascular risk factors in Indian patients undergoing coronary artery bypass surgery. J Assoc Physicians India. 2006;54:371-375.

4. Tai E.s., Emmanuel S.C., Chew S.K., Tan B.Y., Tan c.E. Isolated low HDL cholesterol: an insulin-resistant state only in the presence of fasting hypertriglyceridemia. Diabees. 1999;48:1088-1092.

5. Kulkarni K.R., Markovitz J.H., Nanda N.C., Segrest J.P. Increasd prevalence of smaller and denser 1dl particles in Asian Indians. ArteriosclerThrombVasc Biol. 1999;19:2749-2755.

6. Gupta R., guptha S., Agrawal A., Kaul V., Gaur K., Gupta V.P. Secular Trends in cholesterol lipoproteins and triglycerides and prevalence of dyslipidemias in an urban Indian population. Lipids Health Dis. 2008;740-40.

7. Mahato RV, Gyawali P, Raut PP, Regmi P, Khelanand Ps, Dipendra RP, Gyawali P. Association between glycaemic control and serum lipid profile in type 2 diabetc patients: glycatedhaemoglobin as a dual biomarker. Biomed Res. 20111;22(3);375-380.15

8. Regmi P, Gyawali P, Shrestha R, Sigdel M, Mehta KD, Majhi S. Pattern of dyslipidemia in type-2 diabetic subjects in Eastern Nepal. J Nepal Assoc Med Lab Sci. 2009;10(1):11-13.

9. Krauss RM. Lipids and lipoproteins in patients with type 2 diabetes. Diabetes Care. 2004;27(6);1496-1504.

10. Mooradian AD. Dyslipidemia in type 2 diabetes meliitus. Nat ClinPractEndocrMetab. 2009;5:150-159.

11. smith S, Lali AM. A study on lipid profile leels of diabetics and nondiabetics among Naini region of Allahabad, India. Turk J biochem. 2008;33(4):138-141.

12. Hachem SB, Mooradian Ad. Familial Dyslipidaemias: an overview of generics, pathophysiology and management. Drugs. 2006;66:19491969.

Copyright: () the author(s), 2019. It is an open-access article distributed under the terms of the Creative Commons Attribution License (CC BY 4.0), which permits authors to retain ownership of the copyright for their content, and allow anyone to download, reuse, reprint, modify, distribute and/or copy the content as long as the original authors and source are cited.

How to cite this article: Karan VN, Mohan V. The Association between Serum Lipid Profile and Retinopathy in Type 2 Diabetes Mellitus Patients. Acad. J Med. 2019;2(1):22-25.

DOI: dx.doi.org/10.21276/ajm.2019.2.1.7

Source of Support: Nil, Conflict of Interest: None declared. 